\title{
General Practitioners' Perspectives about Addressing Family Violence with Men from Refugee and Immigrant Backgrounds in Victoria, Australia
}

\author{
Melinda Yunkyo Cho1, Gemma McKibbin'², Mohajer A. Hameed ${ }^{1,3^{*}}$ \\ ${ }^{1}$ Department of General Practice, University of Melbourne, Melbourne, Australia \\ ${ }^{2}$ Department of Social Work, University of Melbourne, Melbourne, Australia \\ ${ }^{3}$ Intergenerational Health Research Group, Murdoch Children’s Research Institute, Melbourne, Australia \\ Email: *mohajer.hameed@mcri.edu.au
}

How to cite this paper: Cho, M. Y., McKibbin, G., \& Hameed, M. A. (2021). General Practitioners' Perspectives about Addressing Family Violence with Men from Refugee and Immigrant Backgrounds in Victoria, Australia. Open Journal of Social Sciences, 9, 304-314.

https://doi.org/10.4236/jss.2021.910022

Received: September 9, 2021

Accepted: October 26, 2021

Published: October 29, 2021

Copyright ( 2021 by author(s) and Scientific Research Publishing Inc. This work is licensed under the Creative Commons Attribution International License (CC BY 4.0).

http://creativecommons.org/licenses/by/4.0/

\begin{abstract}
The purpose of this qualitative article is to explore medical general practitioners' perspectives about addressing family violence with men from refugee and immigrant backgrounds in Victoria, Australia. Ten general practitioners from across Victoria participated in semi-structured interviews. Five major themes relating to general practitioners' perspectives emerged from the thematic data analysis: 1) preference to focus on mothers and children who experience family violence and not men who use family violence; 2) cultural backgrounds acting as a barrier to address family violence; 3 ) family violence as linked to resettlement and mental health concerns; 4) need for a culturally-sensitive and collaborative approach; and 5) broader healthcare systems reform required. Study limitations, implications of findings and recommendations for future research are discussed in terms of broader healthcare systems reform. The authors call for the development of a culturally sensitive and collaborative care system to address family violence with men from refugee and immigrant backgrounds in primary care settings.
\end{abstract}

\section{Keywords}

General Practitioners, Primary Health Care Setting, Family Violence, Men, Refugee and Immigrant Communities, Semi-Structured Interviews

\section{Introduction}

The terms family violence (FV), domestic violence (DV) and intimate partner 
violence (IPV) are often used interchangeably to describe a globally widespread public health and social issue that negatively impacts families, including women, children and men. The World Health Organisation defines IPV as physical, sexual and emotional abuse, as well as controlling behaviours, by an intimate partner (Garcia-Moreno et al., 2012). For the purpose of this paper, the term FV is used.

While both men and women may use FV in relationships, men use FV more frequently and severely, so that women who experience FV are more likely to fear for their lives (World Health Organization, 2013). Men's use of FV is a serious health and social issue for the entire family. In the context of heterosexual relationships, it impacts the woman (AIHW, 2019), any children in the family (Campo, 2015), and the man himself (Walker et al., 2010). Men's use of FV is associated with increased rates of alcohol and substance misuse, depression, suicidal thoughts, stress, anxiety and low self-esteem (Coben \& Friedman, 2002; Oram et al., 2014; Sharps et al., 2001). Men who use FV are also more likely to have experienced childhood abuse, social deprivation and violent victimisation (World Health Organization, 2010). Despite increasing attention to the issue of men's use of FV, progress in reducing its prevalence has been very slow.

Research shows that men who use FV present to general practice for healthcare needs, more often than men who do not use FV (Coben \& Friedman, 2002). This can be for a range of issues including injuries, anxiety or depression. Although men who use FV are often reluctant to seek help or disclose their behaviours, research indicates that it is necessary for general practitioners (GPs) to ask about any potentially abusive behaviour within intimate relationships (Morgan et al., 2014). GPs were viewed as the next most likely source of support for men who use FV after friends and family. Men viewed GPs as a trustworthy source of help due to the reassurance that any disclosed information would remain confidential (Morgan et al., 2014). However, much less is known about how GPs may address the issue of $\mathrm{FV}$ with men from refugee and immigrant backgrounds.

Australia is considered to be a multicultural society with a long history of settling individuals and families fleeing from conflict-torn countries, persecution, war, political and interpersonal violence (Garcia-Moreno et al., 2012; Zannettino, 2012). Men from refugee and immigrant backgrounds are more likely to have experienced these stressors along with challenges associated with cultural transition. Further, they may have to negotiate changes to their role of father and patriarch, as well as different views of gender and family responsibility than those they are accustomed to (Fisher, 2013).

Rees and Pease (2007) found that the factors contributing to refugee and immigrant men's use of FV included the challenges of the resettlement experience, although culturally enforced views of male dominance were also a common theme. The psychosocial issues found to be associated with the resettlement experience included: unemployment and the resulting feelings of worthlessness due to the loss of identity as the "breadwinner" in the family; social isolation due 
to various factors such as fear of racist violence, language barriers, poor social networks and poverty; cultural dissonance and insufficient support to adapt to the Australian culture. Prior trauma experienced by refugee and immigrant families from their country of origin was also found to exacerbate the stresses of resettlement. These factors have been linked with mental health issues that may serve to condone and reinforce men's use of violence in intimate relationships. Consequently, refugee and immigrant families may have higher risk of experiencing and/or using FV (Fisher, 2013; Rees \& Pease, 2007).

Much research in this area has focused on the factors contributing to FV and not health practitioners' perspectives about how to address FV with men from refugee and immigrant backgrounds. This study contributes to filling this gap. The aim of this study was to explore GPs' perspectives about addressing FV with men from refugee and immigrant backgrounds.

\section{Methods}

\subsection{Participants}

The sample consisted of 10 medical general practitioners (GPs) who practiced within primary health care settings across Victoria, Australia. The inclusion criteria included GPs who had clinical experience in providing primary health care with men from refugee and immigrant backgrounds. GPs for whom English was not their first language were also included in this study, since some patients appear to attend clinics where their doctor is of the same cultural background, speaks the same language or is also from a refugee background. However, GPs who did not regularly see patients from refugee or immigrant communities were excluded from the sample. No incentives were offered for participation in this study. Upon request, a summary of the interview findings was provided to individual participants.

\subsection{Procedures and Data Collection}

Following ethics approval $(1,954,245.1)$ by the Medicine and Dentistry Human Ethics Sub-Committee at the University of Melbourne, the sample was recruited via advertisement through VicRen, a practice-based research network of GPs within the Department of General Practice at the University of Melbourne. In addition, GPs were recruited by approaching clinics that had a greater proportion of refugee or immigrant patients and through personal networks. Interested GPs were asked to contact the researchers directly via the contact details in the advertisement flyer. Once participants had expressed their interest to researchers, they were provided with a plain language statement and consent form, which provided details about the study and contact details of a member of the research team.

A date was agreed between the GP participant and the researcher (MYC), at a time and location that was most convenient for the GP. Data was collected via semi-structured telephone or face-to-face interviews. Semi-structured interviews 
with open-ended questions were chosen as the data collection method to enable discussions by sharing experiences, beliefs, opinions and perspectives. Prior to the interview, participants were asked to sign the consent form and complete a brief demographic survey about their gender, age, clinical experience and provision of care for patients from refugee and immigrant backgrounds. Each interview lasted approximately 45-minutes. Discussion topics covered GPs' perspectives and opinions about addressing FV with men from refugee and immigrant backgrounds. The interviews were audio-recorded and transcribed by the researcher (MYC), following confidentiality protocols, including de-identifying participants.

\subsection{Data Analysis}

With the aid of NVivo software, an inductive approach to thematic analysis was used to identify patterns within the data. The method guiding the thematic analysis is set out by Braun \& Clarke (2006) and involves: familiarization with the data; generating initial codes; searching for themes; reviewing of themes; defining and naming themes; and write up of the report. Once initial themes/subthemes were identified, the researcher (MYC) consulted with GM to refine them.

\section{Results}

Ten GPs, based in Victoria, participated in this study. Seven were male and three were female. The age of the GP participants ranged from 32 to 64 years. The GPs' years of clinical experience in Australia ranged from three to 30 years. Four GPs had clinical experience overseas, which ranged from four months to 25 years. Seven GPs were medically trained in Australia, whilst three were trained overseas, in countries such as China, the Republic of Korea and the United Kingdom. The participants' years of experience in providing care for men as well as families from refugee and immigrant backgrounds ranged from three to 20 years.

Five major themes relating to general practitioners' perspectives emerged from the thematic data analysis: 1) preference to focus on mothers and children, not men who use FV; 2) cultural backgrounds acting as a barrier to address FV; 3) FV as linked to resettlement and mental health concerns; 4) need for a culturally-sensitive and collaborative approach; and 5) broader healthcare systems reform required. The statements that have been quoted as evidence are directly from the GP participants.

\section{Preference to focus on mothers and children, not men who use FV}

Most GPs perceived that it was difficult to ask men from refugee and immigrant backgrounds about FV due to an uncertainty about how or when to ask. GPs perceived that there was a risk of appearing judgemental towards the male patient and therefore placing a strain in the doctor-patient relationship. Further, GPs expressed a lack of training and readiness to address FV with their male pa- 
tients regardless of whether they were from an immigrant or refugee background.

"We are atrocious at screening and we are atrocious at picking it up. $P \mathrm{~m}$ sure [identifying FV] is much harder than what we ever thought."

Furthermore, GPs expressed that when they addressed FV with men from refugee and immigrant backgrounds, the appropriate response and referral pathways were not clear and often unknown. Consequently, the GP participants felt that it was much easier to support mothers and their children who experienced FV than addressing the issue with men who use FV.

"I think the referral pathways for perpetrators are not as well known in the GP community. It's a lot easier to think about how you' re going to manage the victim."

Other GPs identified that their role was about prioritising women and children's safety, rather than engaging with men accessing the clinic.

"It's interesting because we were more working on the side of the person experiencing the violence, rather than trying to address the male who was using violence."

Overall, the GP participants were more comfortable with addressing the needs of women and children who are victims of FV rather than the men who are carrying out the abuse and violence.

\section{Cultural background as a barrier to addressing FV}

Most GPs indicated that in some cultures, FV is normalised in marriage and reinforced by patriarchal beliefs and problematic concepts of masculinity. All participants perceived that the cultural backgrounds of refugee and immigrant men could contribute to these barriers when addressing FV.

"The men definitely don't think that what they're doing is wrong. So that's why, I think I find it really, really hard to break that idea."

"Culturally, a lot of men didn't see FV as being an issue ... they thought that using FV was within their rights."

Moreover, GPs perceived that there is a cultural silence and stigma associated with FV that hinders addressing FV within refugee and immigrant communities. GPs perceived that factors such as maintaining family honour and dignity were amongst many that prevented disclosure of FV.

"If immigrant and refugee men are from an 'honour and shame' based culture, where what's morally acceptable is to maintain their family honour and dignity, it's also going to be very difficult for them to confess and to accept that they have a problem in the way that they, as men, deal with family conflicts and violence."

Another barrier involved the lack of a shared definition of FV. One GP reflected on conversations with refugee and immigrant men about FV in which the men did not appear to understand what it constituted.

"It feels purely like a one-way conversation, where you' re telling people what the laws of Australia are and what our ethical values are, but it s just completely lost." 
GPs suggested the need for education and awareness raising about FV with refugee and immigrant families who are settling in Australia.

"The important thing is to try and engage male perpetrators of FV to try and understand their background and what could ve led them to feel that using FV was the only means necessary."

This theme indicated that traditional cultural beliefs about masculinity and patriarchal family structures presented as barriers to the identification of FV used by men from refugee and immigrant backgrounds.

\section{FV as linked to resettlement and mental health concerns}

Nine out of ten GPs perceived that the refugee and immigrant experiences and challenges of resettlement were considerably associated with mental health concerns such as depression, anxiety and post-traumatic stress disorder. The GPs indicated that whilst these stressful experiences and mental health concerns do not justify the use of FV, these issues may be linked.

"Immigrant and refugee men usually come with significant physical and mental health burdens that often contribute to family violence."

The GP participants perceived that the challenges of resettlement in Australia may lead both men and women to feel trapped within difficult circumstances, and hinder help-seeking behaviours. These challenges may include ongoing stress about relatives who may still be in unsafe places overseas; social isolation; racism and discrimination; language barriers; separation from family and friends; and financial issues.

"Family violence is a sense of being trapped ... she can't talk to anybody ... and he's similar ... Immigrant and refugee families would have difficulties with sharing relationship struggles when they're in this new, foreign, isolated community."

Other issues such as financial struggles and unemployment, housing issues and feelings of a loss of cultural identity and uncertainty about the future were also seen to be linked to the use of FV.

"There are much broader issues around employment, education, financial challenges and housing challenges-there are all these other stresses that are going on for these men, and family violence is the tip of the iceberg."

The GPs suggested that some ways in which these issues may be addressed include: referral to psychological and social supports; further education and training for GPs regarding refugee and immigrant mental health and social concerns; and encouraging follow-up consultations for mental health and/or general health consultations with men from refugee and immigrant backgrounds.

\section{Need for a culturally sensitive and collaborative approach}

Nine out of ten participants perceived that providing care and support with male patients who use FV ought to be done in collaboration with cultural community groups and with mental health professionals. Community groups were perceived as an appropriate setting for providing refugee and immigrant men with ongoing support, including education about FV, and for encouraging men 
who use FV to seek help. Some participants indicated that GPs should engage with community groups as part of their care for refugee and immigrant male patients who use FV, although this did not appear to be happening.

"[currently] the bridges between general practice and other community service providers are virtually non-existent."

Other GPs were not aware if such community groups existed.

" $P m$ not sure if there are any specific organisations or [community] groups that deal with male perpetrators of FV."

GPs also viewed that it was appropriate to refer refugee and immigrant men who use FV to men's groups and psychologists who are experienced in cross-cultural counselling and trauma-informed care.

"The outcomes are massively positive when men are referred to a psychologist who will collaborate, build that bridge and take the time to understand all of the complexities."

GPs broadly perceived their role as someone who listens and offers support to refugee and immigrant men who use FV. A long-term, trusting relationship with these male patients was seen to be a key facilitator for men's disclosure of their use of FV.

\section{Broader healthcare systems reform required}

GPs stated that most clinics provide refugee and immigrant families with a one-off health assessment and then require additional payments for further consultations. This was perceived to be due to the lack of financial reimbursement from the healthcare system and limitations of the Medicare system in Australia. GPs who provided care and support for refugee and immigrant families on repeat occasions reported doing so at their own cost.

"Further consultations are not billed as refugee health assessments ... because there is no item number, we'll just do long item numbers for long consults ... There's a huge disincentive for other practitioners to not do this work, because why would you? If there's no extra funding, if there's no recompense, if it s quite emotionally draining and takes up a lot of resources."

Participants indicated the need for broader healthcare system reform with an increase in funds to establish services tailored towards the health needs of refugee and immigrant communities. GPs said that a shift towards more tailored services would allow for longer appointment times, secondary consultations, more efficient interpreter systems and culturally appropriate services that are bulk billed and set up for refugee and immigrant health. Further, it was stated that building connections between GPs and community and social service providers is important.

"We need to be very well connected with settlement agencies and councils. As $G P S$, we need to be out there ... Whilst I do that work now, it is out-of-hours, it's pro bono and dependent on my good will, and quite often, I can't, $P$ ve got my own commitments."

All GPs emphasised the need for clear referral pathways and adequate resources. Many GPs expressed frustration about the lack of a clear referral path- 
way when addressing FV with men from refugee and immigrant backgrounds.

"What services are there for the men to assist them? It's difficult to pry [into use of FV] if GPs don't have a solution or a response... I think having a defined pathway program that I could refer to would be really useful."

The zero-hour contracts by which GPs are currently employed were considered as a barrier for GPs working in collaboration with other GPs, nurses and community service providers when addressing FV with men from refugee and immigrant backgrounds. Overall, GPs perceived that health system reform could enhance referral pathways and adequate resources, as well as collaborative and culturally sensitive care.

\section{Discussion}

This study provided preliminary qualitative research exploring GPs perspectives in addressing FV with men from refugee and immigrant backgrounds. The findings are relevant to the Australian primary health providers addressing FV within culturally and linguistically diverse communities.

The findings showed that generally GPs felt unprepared and uncomfortable to address the issue of FV with men from refugee and immigrant backgrounds. Alternatively, GPs preferred to focus and provide support to mothers and children who experienced FV. The findings that GPs had limited education, training and experience in addressing the issue of FV with male patients were consistent with the previous, yet limited literature. For example, Penti and colleagues (2017) found that whilst GPs acknowledged their role in addressing the issue of FV, they had limited training and knowledge about how and when to address FV with male patients. The participants in this study perceived that they were inadequately equipped to address FV with men from refugee and immigrant backgrounds, due to the lack of knowledge and training about specific referral pathways and available resources. In addition, GPs viewed that they were not supported by the current healthcare system to provide appropriate and ongoing care with men from refugee and immigrant backgrounds who use FV.

Cultural barriers and resettlement issues also complicated the role of GPs in addressing FV with men from refugee and immigrant backgrounds. These barriers are consistent with the broader literature about FV within refugee and immigrant communities. Rees and Pease (2007) emphasised the importance of rethinking settlement policy and practice in Australia, highlighting the issues of social isolation and unemployment that are linked to the refugee and immigrant experiences. Furthermore, issues such as loss of cultural identity and perceived status as the head of the family may contribute to men's feelings of disempowerment and possibly lead to use of FV (Rees \& Pease, 2007).

GPs perceived the need for a more culturally sensitive and collaborative approach when addressing FV with men from refugee and immigrant backgrounds. Previous studies have focused on developing and evaluating collaborative care models in managing and supporting mothers and their children who 
experience FV (Bair-Merritt et al., 2014; Kramer et al., 2012). However, there is very limited research about effective care models with men who use FV (Babcock et al., 2004; Eckhardt et al., 2006; Greaves et al., 2016).

The findings of this study need to be interpreted with caution, given several limitations. The small sample size considerably limits the applicability and generalisability of the findings. This study included GPs working within primary health care settings who had experience supporting refugee and immigrant communities. Therefore, the findings may not generalise to GPs working in other settings (e.g. hospital). Furthermore, whilst this study attempted to recruit GPs from both urban and rural Victoria, all ten GP participants practiced in urban areas. Hence, it is recommended that future research recruit larger samples of GPs from both urban and rural settings to allow for comparing diverse perspectives and opinions about addressing FV with men from immigrant and refugee backgrounds.

\section{Conclusion}

The authors recommend that future research exploring health practitioners' response to $\mathrm{FV}$, focuses on evaluating referral pathways and resources that may be used by GPs when addressing FV with men from refugee and immigrant backgrounds. Furthermore, it is recommended that clinical guidelines and practice frameworks are developed and utilised to train GPs and medical students about how to effectively address and manage FV. Finally, broader healthcare systems reform is required, with increase funding towards the development, implementation and evaluation of culturally-sensitive and collaborative care when addressing FV with refugee and immigrant families.

\section{Acknowledgements}

We gratefully acknowledge contributions made by medical general practitioners in participating in this qualitative research. Also, it is to be noted that this paper was based on first author's fourth year honours thesis and we would like to thank the staff and students at the Department of General Practice, University of Melbourne for their support throughout this research journey.

\section{Conflicts of Interest}

The authors declare no conflicts of interest regarding the publication of this paper.

\section{References}

Australian Institute of Health and Welfare (AIHW) (2019). Family, Domestic and Sexual Violence in Australia: Continuing the National Story 2019. Cat. No. FDV 3. Australian Institute of Health and Welfare.

Babcock, J. C., Green, C. E., \& Robie, C. (2004). Does Batterers' Treatment Work? A Meta-Analytic Review of Domestic Violence Treatment. Clinical Psychology Review, 23, 1023-1053. https://doi.org/10.1016/j.cpr.2002.07.001 
Bair-Merritt, M. H., Lewis-O’Connor, A., Goel, S., Amato, P., Ismailji, T., Jelley, M., Lenahan, P., \& Cronholm, P. (2014). Primary Care-Based Interventions for Intimate Partner Violence: A Systematic Review. American Journal of Preventive Medicine, 46, 188-194. https://doi.org/10.1016/j.amepre.2013.10.001

Braun, V., \& Clarke, V. (2006). Using Thematic Analysis in Psychology. Qualitative Research in Psychology, 3, 77-101. https://doi.org/10.1191/1478088706qp063oa

Campo, M. (2015). Children's Exposure to Domestic and Family Violence: Key Issues and Responses. Journal of the Home Economics Institute of Australia, 22, Article No. 33.

Coben, J. H., \& Friedman, D. I. (2002). Health Care Use by Perpetrators of Domestic Violence. The Journal of Emergency Medicine, 22, 313-317.

https://doi.org/10.1016/S0736-4679(01)00498-X

Eckhardt, C. I., Murphy, C., Black, D., \& Suhr, L. (2006). Intervention Programs for Perpetrators of Intimate Partner Violence: Conclusions from a Clinical Research Perspective. Public Health Reports, 121, 369-381. https://doi.org/10.1177\%2F003335490612100405

Fisher, C. (2013). Changed and Changing Gender and Family Roles and Domestic Violence in African Refugee Background Communities Post-Settlement in Perth, Australia. Violence against Women, 19, 833-847. https://doi.org/10.1177\%2F1077801213497535

Garcia-Moreno, C., Guedes, A., \& Knerr, W. (2012). Intimate Partner Violence. World Health Organization.

Greaves, L., Hemsing, N., \& Poole, N. (2016). Health Interventions Responding to Male Batterers: Are They Ignored and Mistreated? Violence and Gender, 3, 139-142. https://doi.org/10.1089/vio.2015.0041

Kramer, A., Nosbusch, J. M., \& Rice, J. (2012). Safe Mom, Safe Baby: A Collaborative Model of Care for Pregnant Women Experiencing Intimate Partner Violence. The Journal of Perinatal \& Neonatal nursing, 26, 307-316. https://doi.org/10.1097/JPN.0b013e31824356dd

Morgan, K., Williamson, E., Hester, M., Jones, S., \& Feder, G. (2014). Asking Men about Domestic Violence and Abuse in a Family Medicine Context: Help Seeking and Views on the General Practitioner Role. Aggression and Violent Behavior, 19, 637-642. https://doi.org/10.1016/j.avb.2014.09.008

Oram, S., Trevillion, K., Khalifeh, H., Feder, G., \& Howard, L. (2014). Systematic Review and Meta-Analysis of Psychiatric Disorder and the Perpetration of Partner Violence. Epidemiology and Psychiatric Sciences, 23, 361-376.

https://doi.org/10.1017/S2045796013000450

Penti, B., Tran, H., Timmons, J., Rothman, E. F., \& Wilkinson, J. (2017). Physicians' Experiences with Male Patients Who Perpetrate Intimate Partner Violence. Journal of the American Board of Family Medicine, 30, 239-247. https://doi.org/10.3122/jabfm.2017.02.160258

Rees, S., \& Pease, B. (2007). Domestic Violence in Refugee Families in Australia. Journal of Immigrant \& Refugee Studies, 5, 1-19. https://doi.org/10.1300/J500v05n02 01

Sharps, P. W., Campbell, J., Campbell, D., Gary, F., \& Webster, D. (2001). The Role of Alcohol Use in Intimate Partner Femicide. American Journal on Addictions, 10, 122-135. https://doi.org/10.1080/105504901750227787

Walker, D. D., Neighbors, C., Mbilinyi, L. F., O’Rourke, A., Zegree, J., Roffman, R. A., \& Edleson, J. L. (2010). Evaluating the Impact of Intimate Partner Violence on the Perpetrator: The Perceived Consequences of Domestic Violence Questionnaire. Journal of Interpersonal Violence, 25, 1684-1698. https://doi.org/10.1177\%2F0886260509354592 
World Health Organization (2010). Preventing Intimate Partner and Sexual Violence against Women: Taking Action and Generating Evidence. World Health Organization.

World Health Organization (2013). Global and Regional Estimates of Violence against Women: Prevalence and Health Effects of Intimate Partner Violence and Non-Partner Sexual Violence. World Health Organization.

Zannettino, L. (2012). “There Is No War Here; It Is Only the Relationship That Makes Us Scared" Factors Having an Impact on Domestic Violence in Liberian Refugee Communities in South Australia. Violence against Women, 18, 807-828.

https://doi.org/10.1177\%2F1077801212455162 